\title{
Características clínicas, epidemiológicas y evolución de la neumonía nosocomial severa en la unidad de cuidados intensivos
}

\author{
Abel Arroyo-Sánchez ${ }^{a, d}$, Juan Leiva-Goicochea ${ }^{b, d}$, Rosa Aguirre-Mejía ${ }^{c, d}$
}

\section{RESUMEN}

Objetivo: Describir las características clínicas, epidemiológicas, evolución e identificar factores asociados a la mortalidad en pacientes con NNS.

Material y Métodos: Estudio descriptivo de una serie de casos de la Unidad de Cuidados Intensivos (UCI) de un hospital general. Se revisaron las historias clínicas de los pacientes atendidos y que cumplieran los criterios de selección.

Resultados: Cuarenta y un historias clínicas fueron evaluadas. La edad promedio fue de 69.6 años, predominando el género masculino (68.3\%), la NNS fue el motivo de ingreso en $60.9 \%$ y el $95.1 \%$ requirió ventilación mecánica. La estancia hospitalaria previa al diagnóstico fue de 10.0 días, $65.9 \%$ de los pacientes tuvo algún factor de riesgo para organismos multirresistentes, el Clinical Pulmonary Infection Score (CPIS) de ingreso fue 9.3 puntos, los cultivos fueron positivos en $39 \%$ de los casos y de estos el $48.8 \%$ recibieron antibiótico adecuado al cultivo. Los días de estancia en UCI fueron 20.6 días y 20 de las 41 historias correspondieron a pacientes que habían fallecido. Las características clínico epidemiológicas de los fallecidos y sobrevivientes al alta, fueron semejantes. Se realizó un análisis de factores que pudieron estar asociados a mortalidad por NNS encontrando que la edad $\geq 70$ años, la presencia de algún factor de riesgo para microorganismos multidrogorresistente y el CPIS control $\geq 6$ puntos estuvieron asociados a mayor mortalidad; mientras que la adquisición de la NNS en la UCl estuvo asociada a menor mortalidad.

Conclusión: Las características clínicas, epidemiológicas y la evolución de los pacientes con NNS de nuestra UCI fueron semejantes a los descritos en la literatura. Se identificaron tres factores asociados a mortalidad por NNS en la UCI.

Palabras clave: Neumonía Nosocomial. Neumonía Asociada al Ventilador. Neumonía Asociada a los Cuidados de Salud.

\section{Clinical, epidemiological and evolution of severe nosocomial pneumonia in intensive care unit}

\section{ABSTRACT}

Objective: To describe the clinical and epidemiological characteristics, evolution and to identify mortality factors associated in patients with SNP.

Material and Methods: Descriptive study of a serie of cases of the Intensive Care Unit (ICU) of a General Hospital. Medical records of patients which received medical attention and who meet the selection criteria were reviewed

Results: Forty-one clinical records were evaluated. The average age was 69 old, predominantly male (68,3\%). SNP was the reason of admission in $60.9 \%$ and $95.1 \%$ required mechanical ventilation. Hospital stay prior to diagnosis was 10 days, $65 \%$ of patients had some risk factor for multi resistence organisms, CPIS of entry was 9.3 , cultures were positive in $39 \%$ of the cases and of these, $48.8 \%$ received proper antibiotic according to culture results. The days of stay in ICU were 20.6 days and 20 of the 41 medical records were for death patients. The clinical and epidemiological characteristics were similar between death and alive patients. An analysis of factors that could be associated with mortality SNP was made and it was found that for an age $\geq 70$ years, the presence of any risk factor for multidrug resistence organism and control CPIS $\geq 6$ were associated with higher mortality; while acquisition of the ICU was associated to lower mortality.

Conclusions: The clinical, epidemiological characteristics and evolution of patients with SNP in our ICU were similar to those describe in the literature. Three factors associated with mortality in the ICU were identified.

Keywords: Hospital-acquired pneumonia. Ventilator-associated pneumonia. Healthcare-associated pneumonia.

\footnotetext{
${ }^{a}$ Servicio de Cuidados Intensivos e Intermedios, Hospital Víctor Lazarte Echegaray - EsSalud, Trujillo

b Servicio de Medicina Interna, Hospital Víctor Lazarte Echegaray - EsSalud, Trujillo

c Servicio de Diagnóstico por Imágenes, Hospital Víctor Lazarte Echegaray - EsSalud, Trujillo

${ }^{d}$ Facultad de Medicina, Universidad Privada Antenor Orrego, Trujillo
} 


\section{INTRODUCCIÓN}

Las neumonías adquiridas en los hospitales son las más severas y potencialmente fatales. En el año 2004, la Sociedad Americana de Tórax (American Thoracic Society: ATS) y la Sociedad Americana de Enfermedades Infecciosas (Infectious Diseases Society of America: IDSA) aprobaron las Guías para el manejo de adultos con neumonía adquirida en el hospital (neumonía nosocomial: NN), neumonía asociada al ventilador (NAV) y neumonía asociada a los cuidados de salud (NACS) (1).

La NN, NAV y la NACS continúan siendo importantes causas de morbimortalidad a pesar de los avances en la antibioticoterapia, mejores medidas de cuidado en salud y el amplio uso de medidas preventivas(2-7). La NN es definida como aquella que ocurre a partir de las 48 horas de su admisión hospitalaria $(2,3)$, puede ser manejada en sala general o, cuando es severa, requerir manejo en la Unidad de Cuidados Intensivos $(\mathrm{UCl})$. La NAV se refiere a la que ocurre después de 48 horas de inciado el soporte ventilatorio $(3,4)$. La NACS incluye cualquier paciente que ha estado internado en un hospital para pacientes agudos por 2 ó más días dentro de los 90 días de la infección; residentes en un asilo o centros semejantes; receptores recientes de antibioticoterapia endovenosa, quimioterapia o cuidado de heridas dentro de los 30 días de la infección actual; o atendidos en un centro de hemodiálisis hospitalario o privado $(2,3,5)$.

La etiología y el esquema terapéutico recomendado por la guía ATS-IDSA es igual para pacientes con NN o NAV tardías (adquiridas a partir del $5^{\circ}$ día de hospitalización o soporte ventilatorio) o con factores de riesgo para gérmenes multidrogorresistentes (MDR) como son: hospitalización actual mayor de 5 días, antibioticoterapia dentro de los 90 días previos, enfermedad o terapia inmunosupresora, y dos o más de los siguientes criterios: hospitalización $>2$ días en los 90 días previos, residencia en asilo, tratamiento en infusión en su domicilio o cuidados de heridas en los 30 días previos, hemodiálisis crónica > 30 días, miembros de su familia con patógenos MDR; y las NACS (1). En este trabajo de investigación consideramos como Neumonía Nosocomial Severa (NNS) a la NN y NAV tardías o con factores de riesgo para MDR y la NACS $(1,8)$.

El objetivo del estudio fue describir las características clínico epidemiológicas, la evolución e identificar factores asociados a la mortalidad de pacientes con NNS en la UCl.

\section{MATERIAL Y MÉTODOS}

Se realizó un estudio descriptivo de serie de casos, donde se estudiaron las historias clínicas de pacientes con el diagnóstico clínico de Neumonía Nosocomial Severa (Puntaje Clínico de Infección Pulmonar modificado -Clinical Pulmonary Infection Score: CPIS- mayor de 6 puntos e integrada por la NN y NAV tardías o con factores de riesgo para MDR y la NACS) atendidos en la UCI del Hospital Víctor Lazarte Echegaray-EsSalud, Trujillo. Se incluyeron a los pacientes mayores de 18 años que cumplan los criterios de diagnóstico clínico de NNS; excluyéndose a pacientes con otro foco infeccioso adicional a la neumonía, antecedente de tuberculosis pulmonar, gestantes o con estancia hospitalaria en UCI menor de 24 horas.

Se identificaron los casos probables de NNS en base a los diagnósticos de ingreso y egreso anotados en el cuaderno de registro de la $\mathrm{UCl}$, se revisaron las historias clínicas de los pacientes que registraron diagnóstico de "sepsis severa", "shock séptico", "neumonía" e "insuficiencia respiratoria" seleccionando los expedientes que cumplían los criterios de NNS, se llenó la ficha de recolección de datos y se recogieron las siguiente variables: género, edad, diagnóstico de ingreso a la $\mathrm{UCl}$, comorbilidades asociadas, APACHE II y SOFA al ingreso, presencia de disfunción multiorgánica (definida como SOFA $\geq 2$ puntos en 2 ó más órganos), requerimiento de ventilación mecánica al ingreso a la $\mathrm{UCl}$, servicio de adquisición de la NNS, estancia hospitalaria previa al diagnóstico de NNS, factores de riesgo para patógenos MDR (definidos por ATS-IDSA), CPIS diagnóstico o de ingreso, CPIS control a las 72 horas de iniciado el tratamiento, presencia de cultivos positivos, tiempo de inicio de la antibióticoterapia después del ingreso a la UCl (verificado en el kárdex de enfermería), esquema antibiótico empírico inicial adecuado al resultado del cultivo positivo, días de estancia en la $\mathrm{UCl}$ y en el hospital. Así mismo se plantearon factores que pudieron estar asociados a la mortalidad en la $\mathrm{UCl}$, que fueron: género masculino, edad $\geq 70$ años, presencia de alguna comorbilidad, NNS adquirida en nuestra $\mathrm{UCl}$, estancia hospitalaria antes del diagnóstico $\geq 10$ días, presencia de algún factor de riesgo para patógeno MDR, APACHE II $\geq 22$ puntos, SOFA $\geq 10$ puntos, presencia de disfunción multiorgánica al ingreso, requerimiento de ventilación mecánica al ingreso, CPIS diagnóstico $\geq 9$ puntos, CPIS control $\geq 6$ puntos, diagnóstico de NAV tardía o con factor de riesgo para MDR, algún cultivo positivo, uso de antibiótico previo, inicio de la 
antibioticoterapia $\geq$ la $1^{\text {a }}$ hora de su ingreso a la $\mathrm{UCl}$, esquema antibiótico no adecuado al resultado del cultivo positivo.

Los datos fueron procesados con el paquete estadístico SPSS v 15, para luego presentar los resultados en tablas estadísticas de doble entrada de acuerdo a los objetivos planteados.

En el análisis estadístico se hizo uso de la prueba de chi cuadrado $\left(X^{2}\right)$, univariada, de manera alternativa podrá realizarse el análisis de regresión logística. La prueba fue considerada significativa si $p<0.05$.

Se describieron las características clínicas y epidemiológicas del total de pacientes, y se compararon las características de los fallecidos versus vivos al alta de la $\mathrm{UCl}$. Se usó el número de pacientes para las variables cualitativas y las medias para las variables cuantitativas. Finalmente, se compararon los factores previamente definidos y que pudieron estar asociados a la condición del alta de la UCI: fallecido o vivo.

Por ser un estudio descriptivo y de naturaleza anónima no se consideró necesario el consentimiento informado.

\section{RESULTADOS}

Se identificaron 108 historias clínicas con el diagnóstico de ingreso o alta de la $\mathrm{UCl}$ de "sepsis severa", "shock séptico", "neumonía" o "insuficiencia respiratoria"; de las cuales 41 cumplieron los criterios de selección. Predominó el género masculino con el $68.3 \%$ del total de pacientes, con una edad promedio de $69.6 \pm 17$ años. El $60.9 \%$ de los pacientes (25/41) ingresaron con diagnóstico de NNS y el $39.1 \%$ la adquirireron en nuestra unidad. El $46.3 \%$ tuvo alguna comorbilidad al ingreso, siendo la más frecuente la enfermedad pulmonar obstructiva crónica -EPOC- (26.8\%). La disfunción multiorgánica al ingreso estuvo presente en el $41.5 \%$ y requirieron ventilación mecánica el $95.1 \%$ del total de pacientes ingresados. La NNS fue adquirida con mayor frecuencia en los servicios clínicos de nuestro hospital $(18 / 41 ; 43.9 \%)$, seguida por la $\mathrm{UCl}$, servicios quirúrgicos y la adquirida después de recibir atención en los cuidados de la salud. El CPIS inicial (diagnóstico) fue $9.3 \pm 1.3$ puntos y el CPIS control de $4.7 \pm 1.8$ puntos. El $61 \%$ de todos los pacientes tuvieron algún cultivo positivo (25/41) y de ellos en 20 de los 25 (80\%) el antibiótico empírico usado fue adecuado al resultado del cultivo (Tabla 1).

Tabla 1. Características clínico epidemiológicas de los casos y controles de neumonía nosocomial severa.

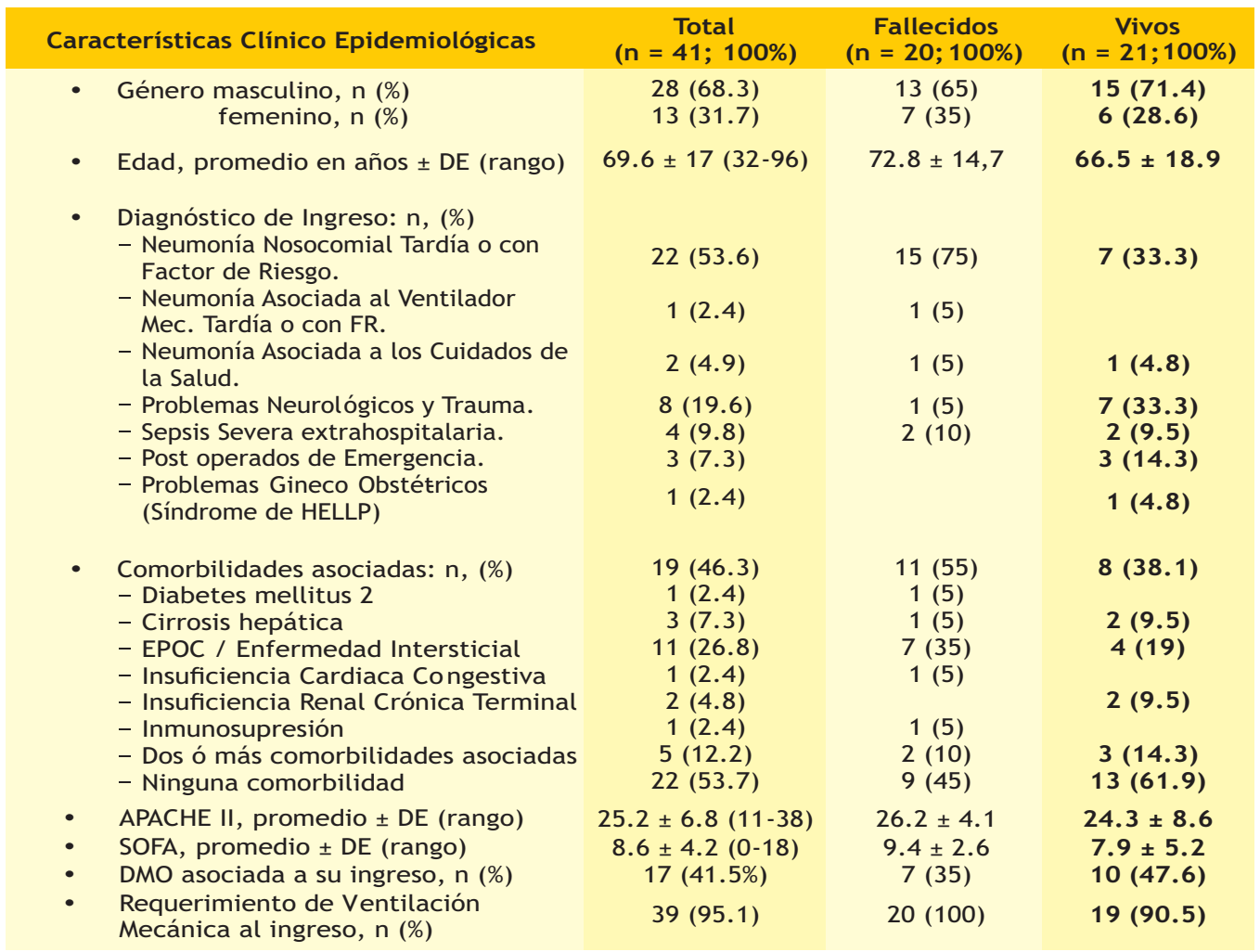


- Lugar de adquisición de la NNS, n (\%): - UCI

- Servicios Clínicos *

- Servicios Quirúrgicos

- Emergencia

- Neumonía Adquirida en los Cuidados de la Salud

- Estancia hospital previa diagnóstico, promedio días $\pm \mathrm{DE}$ (rango)

27 (65.9)

- Dos o más de los siguientes:

a. Hospitalización > 2 días en los 90 días previos.

b. Residencia en asilo.

c. Tratamiento en infusión en su domicilio.

d. Hemodiálisis crónica > 30 días. - Dos ó más Factores de Riesgo

- $\quad$ Tipo de NNS diagnosticada: n, (\%):

- NN tardía o con factores de riesgo para MDR.

- NAV tardía o con facto res de riesgo para MDR.

- NACS.

- CPIS inicial (diagnóstico), promedio \pm DE (rango):

- CPIS control (72 horas después) promedio \pm DE (rango):

- Cultivos positivos: $n,(\%)$

- Secreción bronquial

- Hemocultivo

- Secreción bronquial y hemocultivo positivos

- Tiempo de inicio del antibiót. después del diagnóstico, n (\%)

- Menos de una hora.

- De una a cuatro horas.

- Más de cuatro horas.

- Esquema de antibiótico adecuado para el cultivo, n(\%)

- Días de estancia hospitalaria en la $\mathrm{UCI}$, promedio $\pm \mathrm{DE}$ (rango):

- Días de estancia hospitalaria total, promedio $\pm \mathrm{DE}$ (rango): ***
$16(39.0)$

$18(43.9)$

$4(9.8)$

$1(2.4)$

2 (4.9)

$10.0 \pm 6.2(3-33)$

$14(34.1)$

22 (53.7)

1 (2.4)

$4(9.8)$

$3(15)$
$13(65)$
$2(10)$
$1(5)$
$1(5)$

$13(61.9)$

5 (23.8)

2 (9.5)

1 (4.8)

$10.4 \pm 6.2$

$9.7 \pm 0.4$

17 (85)

$10(47.6)$

3 (15)

$11(52.4)$

15 (75)

7 (33.3)

1 (5)

1 (5)

$3(14.3)$

$10(37.0)$

7 (41.2)

3 (30.0)

$28(68.3)$

15 (75)

13 (61.9)

11 (26.8)

4 (20)

7 (33.3)

2 (4.9)

1 (5)

1 (4.8)

$9.3 \pm 1.3(7-12)$

$9.3 \pm 1.3$

$9.3 \pm 1.5$

$4.7 \pm 1.8(1-9)$

$5.7 \pm 1.9$

$3.8 \pm 1.2$

25 (60.9)

19 (46.3)

$6(14.6)$

11 (55)

9 (45)

2 (10)

14 (66.6)

$10(47.6)$

4 (19)

3 (7.3)

3 (14.3)

18 (43.9)

$18(43.9)$

$5(12.2)$

20 (48.8)

$20.6 \pm 17.6(1-90)$

$16.15 \pm 11.8$

$9(42.8)$

$\begin{array}{lc}8(40) & 10(47.6) \\ 3(15) & 2(9.5) \\ 7(35) & 13(61.9)\end{array}$

$\begin{array}{lr}8(40) & 10(47.6) \\ 3(15) & 2(9.5) \\ 7(35) & 13(61.9)\end{array}$

$\begin{array}{lr}8(40) & 10(47.6) \\ 3(15) & 2(9.5) \\ 7(35) & 13(61.9)\end{array}$

$7(35)$

$24.9 \pm 21.2$

$31.3 \pm 22.7(1-93)$

$19.1 \pm 12.9$

$42.9 \pm 24.1$

* Servicios Clínicos: Incluyen los servicios clínicos del Hospital Lazarte y de otros hospitales de referencia.

** $\quad \mathrm{p}=0.01 ; \mathrm{IC} 95 \% 1.4-27.8$

$* * * \quad p=0.016 ;$ IC $95 \% 11.4-36.1$

DE: Desviación Estándar. FR: Factor de Riesgo.

EPOC: Enfermedad Pulmonar Obstructiva Crónica. APACHE II: Acute Physiology and Chronic Health Evaluation. SOFA: Sequential Organ Faillure Assesment. DMO: Disfunción Múltiple de Órganos. NNS: Neumonía Nosocomial Severa, UCI: Unidad de Cuidados Intensivos, MDR: Multidrogo Resistente, NN: Neumonía Nosocomial. NAV: Neumonía Asociada al Ventilador. NACS: Neumonía Asociada a los Cuidados de Salud. CPIS: Clinical Pulmonar Infection Score.

Veinte de los pacientes habían sido dados de alta en condición de fallecidos (48.8\%) y 21 en condición de vivos (51.2\%). La presencia de factores de riesgo para microorganismos multidrogorresistentes (MDR) estuvieron presentes en el $65.9 \%$ de todos los pacientes y fueron más frecuentes en los fallecidos (85\%; 17 de 20 pacientes) respecto a los vivos (47.6\%; 10 de 21 pacientes) con una diferencia 
estadísticamente significativa $(\mathrm{p}=0.01$; IC 1.4-27.8); de los factores antes mencionados, el más frecuente fue el uso de antibióticos dentro de los 90 días previos al diagnóstico de NNS, encontrado en 15 de los 17 fallecidos y en 7 de los 10 vivos.

Cuando se aplicaron las tablas de $2 \times 2$ para ver si habían factores asociados a mortalidad en los casos de NNS (tabla 2), el análisis bivariante evidenció que la edad $\geq 70$ años ( $p=0.03$; OR: 5.15; IC 95\%: 1.2 23.0), la adquisición de la NNS en la UCI ( $p=0.002$; OR: 0.12; IC 95\%: 0.02 - 0.59), la presencia de algún factor de riesgo para microorganismo MDR $(p=0.01$; OR: 6.23; IC 95\%: 1.4 - 27.8), y el CPIS control $\geq 6$ puntos ( $p<0.001$; OR 3.33; IC 95\%: 1.93 - 5.76) tuvieron diferencia significativa entre los fallecidos y los vivos.

Tabla 2. Factores de riesgo asociados a mortalidad en los pacientes con neumonía nosocomial severa

\begin{tabular}{|c|c|c|c|c|c|}
\hline Factor de Riesgo & $\begin{array}{c}\text { Casos } \\
\text { (20 pac.) }\end{array}$ & $\begin{array}{l}\text { Controles } \\
\text { (21 pac.) }\end{array}$ & $\mathrm{p}$ & OR & IC $95 \%$ \\
\hline Género masculino & 13 & 15 & 0.46 & 0.74 & $0.2-2.8$ \\
\hline Edad $\geq 70$ años & 17 & 11 & 0.03 & 5.15 & $1.2-23$ \\
\hline Alguna comorbilidad asociada & 11 & 8 & 0.22 & 1.99 & $0.6-7.0$ \\
\hline NNS adquirida en UCI & 3 & 13 & 0.002 & 0.11 & $0.02-0.59$ \\
\hline $\begin{array}{l}\text { Hospitalizado antes del } \\
\text { diagnóstico } \geq 10 \text { días }\end{array}$ & 9 & 9 & 0.57 & 0.92 & $0.27-3.15$ \\
\hline $\begin{array}{l}\text { Presencia de algún factor para } \\
\text { microorganismo MDR }\end{array}$ & 17 & 10 & 0.01 & 6.23 & $1.4-27.8$ \\
\hline APACHE II $\geq 22$ puntos & 18 & 14 & 0.08 & 4.5 & $0.81-25.12$ \\
\hline SOFA $\geq 10$ & 11 & 7 & 0.14 & 2.44 & $0.69-8.66$ \\
\hline Presencia de DMO al ingreso & 7 & 10 & 0.31 & 0.59 & $0.17-2.08$ \\
\hline $\begin{array}{l}\text { Requerimiento de Ventilación } \\
\text { Mecánica al ingreso }\end{array}$ & 20 & 19 & 0.26 & 0.49 & $0.35-0.67$ \\
\hline $\begin{array}{l}\text { CPIS inicial o diagnóstico } \geq 9 \\
\text { puntos }\end{array}$ & 13 & 14 & 0.58 & 0.93 & $0.25-3.38$ \\
\hline CPIS control $\geq 6$ puntos & 11 & 0 & $<0.001$ & 3.33 & $1.93-5.36$ \\
\hline $\begin{array}{l}\text { Diagnóstico de NAV tardía o factor } \\
\text { de riesgo MDR }\end{array}$ & 8 & 7 & 0.65 & 1.33 & $0.31-5.78$ \\
\hline Algún cultivo positivo & 11 & 14 & 0.33 & 0.61 & $0.17-2.16$ \\
\hline $\begin{array}{l}\text { Inicio del antibiótico después de la } \\
1^{\text {a }} \text { hora de su ingreso a UCl }\end{array}$ & 11 & 12 & 0.57 & 0.92 & $0.27-3.15$ \\
\hline $\begin{array}{l}\text { Antibiótico inicial no adecuado al } \\
\text { resultado del cultivo }\end{array}$ & 4 & 1 & 0.10 & 7.43 & $0.69-79.96$ \\
\hline
\end{tabular}

NNS: Neumonía Nosocomial Severa, UCl: Unidad de Cuidados Intensivos, MDR: Multidrogo Resistente, APACHE II: Acute Physiology and Chronic Health Evaluation, SOFA: Sequential Organ Faillure Assesment, DMO: Disfunción Múltiple de Órganos, CPIS: Clinical Pulmonar Infection Score.

\section{DISCUSIÓN}

La UCI del Hospital Base Víctor Lazarte Echegaray es una unidad polivalente y de referencia regional para los pacientes con seguro social. Es una unidad cerrada, consta de 6 camas y con médico intensivista las 24 horas del día.

De acuerdo a las historias clínicas que se pudieron recuperar y que cumplían los criterios de selección, la NNS se presentó predominantemente en pacientes del género masculino $(68.3 \%, 28 / 41)$ y adultos mayores de 60 años $(75.6 \% ; 31 / 41)$ con edad promedio $69.6 \pm 17$ años; estos hallazgos fueron semejantes a los descritos por Leroy (9), Soo Hoo (10), Lee (11) y Luna (12), aunque nuestra edad promedio fue mayor a la de otros trabajos (13-16) que estudiaron sólo pacientes con NAV; estas variaciones etáreas dependieron del tipo de $\mathrm{UCl}$ y los criterios de inclusión de la población estudiadas. Semejante a lo descrito por SooHoo (10), Iregui (14), Micek (15) y Kollef (17); la EPOC fue la comorbilidad más frecuente en nuestros pacientes; pero tuvimos una menor incidencia de Insuficiencia Cardiaca comparándonos con los autores antes mencionados y a lo encontrado por Ruiz (18). Cuando se hizo el análisis bivariante, ni el género masculino ni la presencia de alguna comorbilidad fueron factores 
asociados a mayor mortalidad; pero la edad $\geq 70$ años sí se encontró estadísticamente asociada a mayor mortalidad ( $p=0.03$; OR 5.15; IC 95\% 1.2 -23) semejante a lo mencionado por Celis (19) y la Asociación Latino Americana de Tórax (ALAT) (20), aunque para algunos autores la edad no ha sido claramente definida como factor de riesgo de mortalidad de NN (21).

El puntaje promedio de los scores APACHE II (25.24 \pm 6.8 puntos) y SOFA ( $8.63 \pm 4.16$ puntos) así como la presencia de Disfunción Múltiple de Órganos (41.5\%; 17/41) y el requerimiento de ventilación mecánica desde el ingreso a la unidad $(95.1 \% ; 39 / 41)$ nos expresa pacientes con severo compromiso de su estado general y con alto riesgo de morbimortalidad, datos concordantes con la población estudiada; teniendo nuestros pacientes totales el puntaje de APACHE II semejante a los encontrados en los estudios de NAV realizados por Heyland (13) (24.1 \pm 6.2 días), Iregui (14) $(24.6 \pm 8.1$ días $)$ y Micek (15) $(23.0 \pm 9.2$ días), estudios en los cuales se evaluaron pacientes que adquirieron NAV después de ingresar a $\mathrm{UCl}$ y no fue exclusivamente severa. La presencia de disfunción múltiple de órganos en nuestro estudio fue mayor a la descrita por Illodo (22) $(37.7 \%, 26 / 69)$. Cuando se hizo el análisis bivariante para detectar factores asociados a mortalidad, el APACHE II > 22 puntos, el SOFA > 10 puntos, la presencia de DMO o el requerimiento de ventilación mecánica no fueron significativos lo que nos orientó a buscar otros factores asociados a mortalidad.

El diagnóstico de ingreso a nuestra $\mathrm{UCl}$ fue de NNS en 25 de los 41 pacientes totales $(60.9 \%)$ siendo más frecuente en el grupo de los fallecidos 17/20 (85\%) comparada con los vivos $8 / 21$ (38\%). Es importante mencionar que 14 de los 25 pacientes (56\%) adquirieron la NNS fuera de nuestro hospital (policlínicos, otros hospitales y clínicas privadas) y fueron referidos a nosotros con el diagnóstico de NNS; algunos estudios publicados sobre NAV, excluyen a los pacientes con diagnóstico previo de NAV $(13,14,22)$; pero en nuestro estudio, al igual que el de Ruiz (18), se trató de reflejar la realidad de nuestra unidad.

La adquisición de la NNS en la $\mathrm{UCl}$ fue un factor asociado a una menor mortalidad $(\mathrm{p}=0.002$; OR: 0.115; IC 95\%: 0.02 - 0.59) esto probablemente se deba a un diagnóstico clínico e inicio del tratamiento antibiótico empírico más precoz, con una orientación microbiológica de la flora predominante en nuestro unidad; a diferencia de las infecciones adquiridas fuera de la $\mathrm{UCl}$ y, con mayor razón, aquellas adquiridas fuera de nuestro hospital. Esto es difícil de comparar, pues en la mayoría de los trabajos realizados se excluyen los pacientes con diagnóstico previo de Neumonía Nosocomial.

Los días de estancia hospitalaria previa al diagnóstico han sido postulados como un factor de riesgo de morbimortalidad en la NAV en varios estudios y revisiones, pero esto no ha sido estudiado en pacientes con NNS. La estancia hospitalaria previa al diagnóstico de NNS del total de pacientes en nuestro estudio fue de 10 $\pm 6,2$ días, sin existir diferencia significativa entre los fallecidos y vivos; nuestra estancia hospitalaria previa al diagnóstico de NNS fue menor a la descrita por Leroy (9)y Soo Hoo (10)(15.4 \pm 12.2 y $20.4 \pm 16.6$ días respectivamente), pero mayor a la encontrada por Heyland (13) (7 días), Iregui (14) (7.1 \pm 3.9 días), e lllodo (22)(5.3 días).

La presencia de algún factor de riesgo para microorganismos MDR se encontró en el $65.7 \%$ de los pacientes totales $(27 / 41)$ y estuvo presente en el $85 \%$ de los fallecidos $(17 / 20)$ a diferencia del $47.6 \%$ de los vivos $(10 / 21)$ asociandose a mayor mortalidad por NNS ( $p=0.01$; OR: 6.23; IC 95\%: 1.4 - 27.8). La relación entre microorganismos MDR y mortalidad ha sido documentada en varios estudios $(13,23)$, y los factores de riesgo para MDR en NNS son los descritos en la Guías de manejo de neumonía intrahospitalaria de la ATS $^{1}$ y de la ALAT (20). Es de remarcar que el principal factor de riesgo para MDR en nuestro estudio fue el uso de antibióticos dentro de los 90 días previos, lo que podría reflejar un problema actual de la práctica clínica en las diferentes instituciones de salud de nuestro país y la necesidad de generar unidades que garanticen el uso racional y controlado de los antimicrobianos.

Las medias del CPIS al ingreso (usado en nuestro estudio para el diagnóstico clínico de NNS) y el CPIS control (72 horas después del diagnóstico e iniciado el tratamiento antimicrobiano) no fueron diferentes estadísticamente entre los fallecidos y controles. Nuestros CPIS de ingreso fue mayor al reportado por Luna et al en pacientes con NAV (24); a pesar de esto, al hacer el análisis bivariante, se evidenció que un CPIS de ingreso mayor o igual a 9 puntos no estuvo asociado estadísticamente a los fallecidos, pero un CPIS control mayor o igual a 6 puntos si estuvo asociado a mayor mortalidad $(\mathrm{p}<0.001$; OR 3.33; IC 95\% 1.93 -5.36). Desde la implementación del CPIS por Pugin et al en 1991, su utilidad como método de diagnóstico clínico es recomendado por las guías de tratamiento de neumonía nosocomial $(1,20,23)$ y 
algunas revisiones de expertos $(6,21,25)$, pues la probabilidad de realizar procedimientos diagnósticos invasivos no esta disponible las 24 horas del día en la mayoría de las $\mathrm{UCl}$ del mundo. Por otro lado la utilidad del CPIS en el proceso de desescalamiento también está recomendada en las guías y la no disminución de su puntaje inicial o su elevación nos orienta a pensar en una cobertura inadecuada $(24,26)$.

La presencia de algún cultivo positivo, el tiempo de inicio del antibiótico después de la primera hora del diagnóstico clínico de NNS, el esquema antimicrobiano mono o triple, y el antibiótico inicial no adecuado al resultado del cultivo positivo, no estuvieron asociados significativamente a los fallecidos por NNS; hallazgos diferentes a los descritos por otros autores $(1,6,20,23,25,27)$.

El tiempo de estancia hospitalaria total fue diferente entre los fallecidos y los vivos ( $p=0.016$; IC 95\%: 11.4 - 36.1), siendo $19.1 \pm 12.9$ días para los primeros y $42.9 \pm 24.1$ días para los segundos. Esta corta estancia en los fallecidos puede haber estado relacionada a la mortalidad de los pacientes secundario a su gravedad. Lo encontrado por nosotros fue semejante a lo descrito por Heyland (13) quien encontró 4.7 días adicionales de estancia hospitalaria en los pacientes con NAV sobrevivientes vs. 2.6 días adicionales en los pacientes con NAV que fallecieron ( $p<0.001$; IC 1.5 7.0 días) e Iregui (14) con estancia hospitalaria en los pacientes con NAV sobrevivientes 27.9 días vs. 22.3 días de los fallecidos $(p=0.009)$. Pero, opuesto a lo descrito por Illodo (22), quien estudió pacientes con NAV encontrando 5 días de estancia en $\mathrm{UCl}$ en los fallecidos y 3.4 días en los sobrevivientes, y a lo descrito por Heyland (13) en una conferencia consenso de expertos sobre $\mathrm{NN}$ adquirida en $\mathrm{UCl}$.

Nuestros hallazgos sugieren que los pacientes que ingresaron a nuestro servicio con el diagnóstico previo de de NNS tuvieron mayor mortalidad y es probable que esto sea debido a: el retraso en el diagnóstico, el inicio de la cobertura antimicrobiana tardía (mayor de 24 horas) o a la cobertura inapropiada al no conocer la flora microbiana del servicio donde se adquirió la infección.

El presente trabajo tiene las limitaciones propias de un estudio retrospectivo, el pequeño tamaño de la muestra y la falta de evidencia microbiológica en todos los pacientes participantes, mismas que se pueden evitar en un estudio prospectivo de este tipo de pacientes.
En conclusión, el perfil clínico, epidemiológico y la evolución de los pacientes de nuestro estudio es semejante a la descrita en la literatura mundial. La edad $\geq 70$ años, la presencia de algún factor de riesgo para microrganismo MDR y el CPIS control $\geq 6$ puntos estuvieron asociados a la mortalidad de los pacientes con NNS en la UCl.

De acuerdo a nuestros hallazgos recomendamos iniciar el tratamiento antimicrobiano empírico racional lo más precoz posible, una vez que se sospecha la NNS, incluso antes de que el paciente pueda ingresar a $\mathrm{UCI}$, implementar un sistema de control y uso racional de antimicrobianos a nivel hospitalario, y finalmente replantear el manejo integral del paciente con CPIS control $\geq 6$ puntos, como la etiología y la aparición de injurias adicionales.

\section{REFERENCIAS BIBLIOGRÁFICAS}

1. American Thoracic Society. Guidelines for the management of adults with hospital-acquired, ventilator-associated, and healthcare-associated pneumonia. Am J Respir Crit Care Med 2005; 171: 388-416.

2. Niederman MS.Guidelines for the management of respiratory infection: why do we need them, how should they be developed, and can they be useful? Curr Opin Pulm Med 1996;2:161-5.

3. Craven DE, Kunches LM, Kilinsky V, Lichtenberg DA, Make BJ, McCabe WR. Risk factors for pneumonia and fatality in patients receiving continuous mechanical ventilation. Am Rev Respir Dis 1986; 133:792-796.

4. Tablan OC, Anderson LJ, Besser R, Bridges C, Hajjeh R, Healthcare Infection Control Practices Advisory Committee, Centers for Disease Control and Prevention. Guidelines for preventing health-care-associated pneumonia, 2003: recommendations of the $\mathrm{CDC}$ and the Healthcare Infection Control Practices Advisory Committee. MMWR Recomm Rep 2004;53(RR-3):1-36.

5. Hutt E, Kramer AM. Evidence-based guidelines for management of nursing home-acquired pneumonia. J Fam Pract 2002;51:709-716.

6. Chastre J, Fagon JY. Ventilator-associated pneumonia. Am J Respir Crit Care Med 2002;165:867-903.

7. Richards MJ, Edwards JR, Culver DH, Gaynes RP. Nosocomial infections in medical ICUs in the United States: National Nosocomial Infections Surveillance System. Crit Care Med 1999;27:887-892.

8. Eggimann P, Hugonnet S, Sax H, Touveneau S, Chevrolet JC, Pittet D. Ventilator-associated pneumonia: caveats for benchmarking. Intensive Care Med 2003;29:2086-2089.

9. Leroy O, Jaffré S, d'Escrivan T, Devos P, Georges H, Alfandari S. Hospital-Acquired Pneumonia. Risk factors for 
antimicrobial-resistant causative pathogens in critically ill patients. Chest 2003;123:2034-2042.

10. Soo Hoo G, Wen E, Nguyen T, Bidwell Gotees M. Impact of clinical guidelines in the management of severe HospitalAcquired Pneumonia. Chest 2005;128:2778-2787.

11. Lee S, Hua C, Yu T, Shieh W, See L. Risk factors of mortality for nosocomial pneumonia: importante of inicial anti-microbial therapy. Int J Clin Pract 2005;2005:39-45.

12. Luna C, Aruj P, Niederman M, Garzon J, Violi D, Prignoni A et al. Appropiateness and delay to initiate therapy in ventilador-associated pneumonia. Eur Respir J 2006;27:158 164.

13. Heyland DK, Cook DJ, Griffith L, Keenan SP, Brun-Buisson C, Canadian Critical Trials Group. The attributable morbidity and mortality of ventilator-associated pneumonia in the critically ill patient. Am J Respir Crit Care Med 1999;159:1249-1256.

14. Iregui M, Ward S, Sherman G, Fraser VJ, Kollef MH. Clinical importance of delays in the initiation of appropriate antibiotic treatment for ventilator-associated pneumonia. Chest 2002;122:262-268.

15. Micek S, Ward S, Fraser V, Kollef M. A randomized controlled trial o fan antibiotic discontinuation policy for clinically suspected ventilator-associated pneumonia. Chest 2004;125:1791-1799.

16. Kollef M, Morrow L, Niederman M, Leeper K, Anzueto A, BenzScott L et al. Clinical characteristics and treatment patterns among patients with ventilator-associated pneumonia. Chest 2006;129:1210-1218.

17. Kollef M, Shorr A, Tabak Y, Gupta V, Liu L, Johannes R. Epidemiology and outcomes of health-care-associated pneumonia. Results from a large US database of culturepositive pneumonia. Chest 2005; 128: 3854 - 3862.

18. Ruiz M, Guerrero J, Romero C. Etiología de la neumonía asociada a ventilación mecánica en un hospital clínico. Asociación con comorbilidad, uso previo de antimicrobianos y mortalidad. Rev Chil Infect 2007;24(2):131-136.

19. Celis R, Torres A, Gatell JM, Almela M, Rodriguez-Roisin R, Agusti-Vivaldi A. Nosocomial pneumonia.A multivariate analysis of risk and prognosis. Chest 1988;93(2):318-324.

20. Luna C, Monteverde A, Rodríguez A, Apezteguía C, Zabert G, Ilutovich $\mathrm{S}$, et al. Neumonía Intrahospitalaria: guía clínica aplicable a Latinoamérica preparada en común por diferentes especialistas. Arch Bronconeumol 2005;41:439-456.

21. Arancibia F, Ruiz M. Neumonía asociada a ventilación mecánica: Enfoque actual. Rev Chil Med Intensiva 2004;19(2):63-72.

22. Illodo O, Cristo V, Silva S, Gutierrez L, Fundora E, Cruz C. Factores de riesgo relacionados con la mortalidad en la neumonía asociada la ventilación. Rev Cub Med Int Emerg 2003;2:69-75.

23. Rotstein C, Evans G, Born A, Grossman R, Light B, Magder S et al. Clinical practice guidelines for hospital-acquired pneumonia and ventilator-associated pneumonia in adults. Can J Infect Dis Med Microbiol 2008;19(1):19-53.
24. Luna CM, Blanzaco D, Niederman MS, Matarucco W, Baredes NC, Desmery P, Palizas F, Menga G, Rios F, Apezteguia C. Resolution of ventilator-associated pneumonia: prospective evaluation of the clinical pulmonary infection score as an early clinical predictor of outcome. Crit Care Med 2003;31:676-682.

25. Koening S, Truwitt J. Ventilator-associated pneumonia: diagnosis, treatment and prevention. Clin Microb Rev 2006;19(4):637-657.

26. Dennesen PJ, Van der Ven AJ, Kessels AG, Ramsay G, Bonten MJ. Resolution of infectious parameters after antimicrobial therapy in patients with ventilator-associated pneumonia. Am J Respir Crit Care Med 2001;163:1371-1375.

27. Torres A, Aznar R, Gatell JM, Jimenez P, Gonzalez J, Ferrer A et al. Incidence, risk, and prognosis factors of nosocomial pneumonia in mechanically ventilated patients. Am Rev Respir Dis 1990;142(3):523-528.

\section{Fuentes de financiamiento}

Este articulo ha sido financiado por los autores.

\section{Conflictos de interés}

Los autores declaran no tener ningún conflicto de interés.

\section{Correspondencia:}

Abel Salvador Arroyo Sánchez

Dirección: Jr. Grau 372, Cercado, Trujillo, Perú

Teléfonos: 044-288751

Correo electrónico: abelsalvador@yahoo.com

Recibido: 24 de enero de 2016 Aprobado: 15 de febrero de 2016 